International Journal of Pure and Applied Mathematics

Volume 109 No. 4 2016, 899-909

ISSN: 1311-8080 (printed version); ISSN: 1314-3395 (on-line version)

url: http://www.ijpam.eu

doi: $10.12732 /$ ijpam.v109i4.13

\title{
RADON-NIKODYM PROPERTY, KADEC-KLEE PROPERTY, AND ROTUND NORM
}

\author{
Gaj Ram Damai ${ }^{1}$, Prakash Muni Bajracharya ${ }^{2}$, Yongjin $\mathrm{Li}^{3} \S$ \\ ${ }^{1}$ Siddhnath Science Campus (T.U.) \\ Mahendranagar, NEPAL \\ Central Department of Mathematics (T.U.) \\ Kirtipur, Kathmandu, NEPAL \\ ${ }^{3}$ Department of Mathematics \\ Sun Yat-Sen University \\ Guangzhou, 510275, P.R. CHINA
}

\begin{abstract}
The aim of this paper is to give several well-known results of Banach spaces possessing, the Radon-Nikodym property. It is interesting to see how this property can be described in terms of Asplund spaces, spaces admitting KKP, and rotund norm. We study nearly about an open problem [1] mentioned below.
\end{abstract}

AMS Subject Classification: 46B22, 46B20

Key Words: Asplund spaces, Kadec-Klee property, Rotund norm, locally uniform convex norm, slices

\section{Introduction}

The Radon-Nikodym property (RNP) for Banach spaces, which led to a remarkable theory developed largely between 1965 and 1980. The theory concerned with RNP is an impressive example. We have a fascinating interplay between analytic, geometric, and operator theoretic aspects. This property viewed from a purely geometric perspective [10].

Received: $\quad$ August 12, 2016

Revised: $\quad$ September 12, 2016

Published: $\quad$ October 7, 2016

${ }^{\S}$ Correspondence author (c) 2016 Academic Publications, Ltd. url: www.acadpubl.eu 
The RNP of a Banach space has developed many geometrical properties like; extreme point, denting point, exposed point, dentability, slices, Kadec Klee property (KKP), and strictly convexity (rotundity) norm which has been related to analytic concepts. RNP intimately connected with extreme point structure.

Radon-Nikodym property has attracted the attention of many researchers (see [4]) for more details on the RNP). It is clear that the Radon-Nikodym property is invariant under renorming and isomorphic. Fabian and Godefroy proved that every dual space with RNP has an equivalent locally uniformly rotund norm, but for non-dual spaces, the question remains open [17].

After M. Talagrand it is proved in $[21]$ that $C\left(\left[0, \omega_{1}\right]\right)$, where $\omega_{1}$ is the first uncountable ordinal, has an equivalent Frechet differentiable norm, but does not admit any equivalent norm with a strictly convex dual norm, R. Haydon answered negatively the second question mentioned in [11] by constructing a scattered compact space $\mathrm{K}$ such that $C(K)$ does not admit a Gateaux differentiable renorming, nor a strictly convex renorming.

On the other hand R. Deville [6] proved that if $K$ is a scattered compact space and if its $\omega_{1}$ Cantor derived set $K\left(\omega_{1}\right)$ is empty, then $C(K)$ admits a Frechet differentiable renorming. Moreover R. Haydon and C. A. Rogers [11] proved that, under the same assumptions, $C(K)$ admits an equivalent LUC norm.

We say that a Banach space $X$ has Kadec-Klee property (KKP) if the norm topology and weak topology coincide on the unit sphere $S(X)$. It is also called weak Kadec-Klee property. Weak Kade-klee norms play an important role in Banach Space theory and its applications. It is important in renorming theory to make an equivalent Kadec-Klee norms of some classical Banach Spaces. Authors use KK to show that every non-reflexive space can be renormed that there is norm 1 projection of $X^{* *}$ to $X$. In particular, every non reflexive space can be so renormed that it is not isometric to any dual space. Kadec-Klee property is closely related to the other notions in which the linear structure of Banach space is not involved, one of these is $\sigma$-fragment ability. As is well known, KK property was first established J. Radon and subsequently by F. Rieesz who showed that the classical $L_{p}$ spaces, $1<p<\infty$, have the KK property. Although the space $L_{1}[0,1]$ with Lebesgue measure fails to have KK property. Riesz showed that each of the $L_{p}$ spaces, $1 \leq p<\infty$ has the property that each sequence on the unit sphere that converges almost everywhere converges also in norm. A complete reference on Asplund spaces and spaces with the RNP is found in [4]. 


\section{Notations and Terminologies}

In the sequel, $(X,\|\cdot\|)$ is the real Banach space with norm $\|\cdot\| ; S(X)$ is the unit sphere in $X,\left(X^{*},\|\cdot\|^{*}\right)$ is the dual space of $X ; S\left(X^{*}\right)$ is the unit sphere of $X^{*} ; B(X)$ is the unit ball of $X ; B\left(X^{*}\right)$ is the unit ball of $X^{*} ; \Rightarrow, \rightarrow, \neg$ are the strong, weak, and $w^{*}$-convergence of the sequences respectively, and short forms like: NCCB, RNP, KKP, R stand for non-empty closed convex bounded subset of $X$, Radon-Nikodym property, Kadec-Klee property, Rotund (Strictly convex ) norm respectively.

First, let us recall some definitions.

1.1 A Banach space $X$ has Radon-Nikodym property (RNP) if every bounded closed convex subset of $X$ has slices (i.e. a non-empty intersection with a halfspace) of arbitrarily small diameter (see [5], [9], p. 123), i.e. every bounded subset is sliceable. The whole space is not sliceable, so restriction of bounded set is essential (see [6], [8]).

1.2 We say that a Banach space $X$ has Kadec-Klee property (KKP) or $X$ has KK norm if every sequence $\left\{x_{n}\right\}$ in $\mathrm{X}$ converges weakly to some $x \in X$ and $\lim _{n \rightarrow \infty}\left\|x_{n}\right\|=\|x\|$ implies $\lim _{n \rightarrow \infty}\left\|x_{n}-x\right\|=0$. Kadec-Klee property is also called weak Kadec-Klee property.

1.3 A norm on a Banach space $\mathrm{X}$ is said to be Rotund $(R)$ or Strictly convex if for all $x, y \in S(X),\|x+y\|=2$ implies $x=y[6]$.

1.4 A Banach space $X$ is said to be Asplund space if every separable subspaces of $X$ has separable dual.

1.5 A subspace $Y$ of $X$ is complemented in $X$ if there is a projection $P$ : $X \rightarrow X$ such that $P(X)=Y$ and $\|P\|<\infty$.

1.6 A Banach space $X$ is said to have Krien-Milman property if every bounded closed convex set in $X$ has an extreme point. Equivalently, every bounded closed convex set in $X$ is the closed convex hull of the set of all extreme point.

Remark: (1) It is given in [17] that every space with RNP has KMP. Is every KMP imply RNP? The result is true for dual spaces and, for the notion of preserved extreme point of $B\left(X^{*}\right)$ if there is an element $x \in S(X)$ such that for all $\varepsilon>0, x$ determines a slice of $B\left(X^{*}\right)$ containing $f$ and is of diameter less than $\varepsilon>0$.

(2) Strongly exposed point is denting point, but converse is not true even in finite dimensional spaces.

1.7 We say that a point $x \in S(X)$ is called a denting point of $B(X)$ if for all $\varepsilon>0, x$ is contained in a slice of $B(X)$ of diameter less than $\varepsilon>0$. Equivalently, a point belonging to slices of arbitrarily small diameter is called 
denting point.

1.8 A point $x \in A$ is called exposed point of $A$ if there is $f \in X^{*}$ such that $f(x)=\sup f(A)$ and for all $x_{0} \in A$ with $f\left(x_{0}\right)=\sup f(A)$ we have $x=x_{0}$.

1.9 A point $x \in A$ is called strongly exposed point of $A$ if there is $f \in X^{*}$ such that $f(x)=\sup f(A)$ and for all sequence $\left\{x_{n}\right\} \subseteq X$ with $\lim _{n \rightarrow \infty} f\left(x_{n}\right)=$ $\sup f(A)$ imply $x_{n} \rightarrow x$.

1.10. A slice of $A \subseteq X$ is a nonempty subset of the form

$S(A, f, \alpha)=\left\{x \in A: f(x)>\sup f(A)-\alpha, f \in X^{*}, \alpha>0\right\} .[20]$

Next, some examples were given in the following:

1. All Hilbert spaces have RNP.

2. The reflexive spaces and separable duals have RNP. These criteria apply to all $L_{p}$ 's with $1<p<\infty$ and to $l_{1}$.

3. The spaces $c_{0}, l_{\infty}, C[a, b], L_{\infty}[a, b]$, and $L_{1}[a, b]$ are counter examples.

4. For $c_{0}, l_{1}=c_{0}^{*}$, and $l_{\infty}=l_{1}^{*}$, RNP behaves badly under passage to duals or pre duals.

5. Since reflexive Banach spaces are isomorphic to their second dual, they have the RNP .

6. Duals of every smooth spaces possess RNP [8].

7. All WCG spaces possess the RNP [8].

8. A Banach space possessing RNP if its unit ball has RNP.

\section{The Open Problem about Radon-Nikodym Property}

\section{Now, We face the following open problem: [1]}

If the Banach space $X$ has Radon-Nikodym property, does it follow that $X$ has an equivalent weak Kadec-Klee norm? Does it have an equivalent strictly convex norm? Is it true that $X$ admits an equivalent LUR norm?

\section{Some results were known:}

The Radon-Nikodym property carries over to closed subspaces. There is even a remarkable converse: if all separable closed subspaces of a Banach space have the Radon-Nikodym property, then so does the whole space[15].

Theorem 3.1. [3, 5] A Banach space $X$ has RNP iff every non-empty closed convex bounded subset $F$ of $X$ has at least one weakly denting point.

Corollary 3.2. For a Banach space $X$, the following are equivalent:

(1) $X$ has RNP.

(2) Every closed convex bounded subset of $X$ is the closed convex hull of its weakly exposed points (strongly exposed point). 
(3) Every NCCB subset of $X$ has at least one weakly exposed point (denting point).

The central result of the geometry says that the RNP of a Banach space can be characterized by the following equivalent conditions:

1. A Banach space $X$ has RNP if and only if every bounded subset if $X$ is dentable ([5], p.123).

2. Let $X$ be a Banach space such that there is a separable subspace $Y$ of $X$ such that $Y^{*}$ is not separable (i.e. $X^{*}$ does not have RNP), then there is a separable subspace $Z$ of $X^{*}$ such that $Z$ is not isomorphic to a subspace of a separable conjugate space.

3. A convex set $K$ has RNP if and only if each non empty subset of $K$ has non empty open slices with arbitrarily small diameter.

4. A Banach space $X$ possessing RNP if and only if each equivalent norm on the unit ball is sliceable [6].

5. Every space with RNP has subspace isometric to a dual space.

Theorem 3.3. [20] If $X^{*}$ has Radon-Nikodym property then $X$ is an Asplund space.

Proof. Assume that $X^{*}$ has RNP. We have to show $X$ is Asplund space. If possible, assume that $X$ is not Asplnd space. Then from Namioka and Phelps result which is asserted as "Every $w^{*}$ dentability of $w^{*}$-compact convex subsets of the dual space $X^{*}$ characterizes Asplund spaces". So by this result $X$ is not Asplund implies there is $w^{*}$ compact set $C \subseteq X^{*}$ which is not $w^{*}$-dentable. Let $B\left(X^{*}\right)$ be the unit ball of a dual space $X^{*}$. Then the $w^{*}$-closed symmetric convex body $D \equiv B\left(X^{*}\right)+(C-C)$ is also not $w^{*}$ dentable. Hence we consider $B\left(X^{*}\right)$ is not $\mathrm{w}^{*}$ - dentable. So by definition of $\mathrm{w}^{*}$ dentability there is an $\varepsilon>0$ such that for all $x \in S(X)$ there are sequences $\left\{f_{k}\right\},\left\{g_{k}\right\} \subseteq B\left(X^{*}\right)$ with $f_{k}(x), g_{k}(x) \rightarrow 1$ while $\left(f_{k}-g_{k}\right)\left(t_{k}\right)>\varepsilon$ for some $t_{k} \in S(X)$. Let $X_{1}$ be any separable subspace of $X$ and choose a sequence $\left\{x_{n}^{1}\right\}$ which is dense in $S\left(X_{1}\right)$. From above for all $x_{n}^{1}$ there is $\left\{f_{n_{k}}^{1}\right\},\left\{g_{n_{k}}^{1}\right\} \subseteq B\left(X^{*}\right)$ with $f_{n_{k}}^{1}\left(x_{n_{k}}^{1}\right), g_{n_{k}}^{1}\left(x_{n}^{1}\right) \rightarrow 1$ and $x_{n}^{1} \in S(X)$ with $\left(f_{n_{k}}^{1}-g_{n_{k}}^{1}\right)\left(t_{n_{k}}^{1}\right)>\varepsilon$.

Let $X_{2} \equiv \overline{\operatorname{span}}\left(X_{1} \cup t_{n_{k}}^{1}\right)$ then by repeated application, we obtain separable subspaces $X_{1} \subseteq X_{2} \subseteq \cdots \subseteq X_{l} \subseteq X_{l+1} \subseteq \cdots$. Define $X_{0}=\overline{\bigcup X_{l}}$. Then we have to show $B\left(X_{0}^{*}\right)$ is not $w^{*}$ - dentable. As $X^{*}$ has the RNP, is separable. Because if $X^{*}$ has RNP then every separable subspace of $\mathrm{X}$, has separable dual. Since $\bigcup X_{l}$ is dense in $X_{0}$, for all positive sequence $\delta_{m} \searrow 0$ and any $x_{0} \in X_{0},\left\|x_{0}\right\|=1$, there is a norm 1- sequence $\left\{x_{m}\right\}$, i.e. each term of this sequence has norm 1. where $x_{m} \equiv x_{n}^{l}$ for some $n$ and $l$, such that $\left\|x_{0}-x_{m}\right\|<\frac{\delta_{m}}{2}$. From the 
construction there are functionals $f_{m} \equiv f_{n_{k}}^{l}$ and $g_{m} \equiv g_{n_{k}}^{l}$ in $B\left(X^{*}\right)$ where $k$ is large enough that $f_{m}\left(x_{m}\right) \equiv f_{n_{k}}^{l}\left(x_{n}^{l}\right)>1-\frac{\delta_{m}}{2}$ and

$$
g_{m}\left(x_{m}\right) \equiv g_{n_{k}}^{l}\left(x_{n}^{l}\right)>1-\frac{\delta_{m}}{2} .
$$

Thus

$$
\begin{gathered}
f_{m}\left(x_{0}\right)=f_{m}\left(x_{0}-x_{m}\right)+f_{m}\left(x_{m}\right) \\
>\left\|f_{m}\right\| \cdot\left\|x_{0}-x_{m}\right\|+1-\frac{\delta_{m}}{2} \\
>-\frac{\delta_{n}}{2}+1-\frac{\delta_{m}}{2} .
\end{gathered}
$$

Hence $f_{m}\left(x_{0}\right)>1-\delta_{m}$ and similarly, $g_{m}\left(x_{0}\right)>1-\delta_{n}$.

But for all $m,\left\|\left.\left(f_{m}-g_{m}\right)\right|_{X_{0}}\right\| \geq\left(f_{m}-g_{m}\right)\left(t_{n_{l}}\right)>\varepsilon$ a contradiction, it is due to the fact that $X$ is not Asplund. Thus it forces us $X$ is Asplund.

A result of Uhl (1972, p. 114 within [10]) and Stegall (1975, p. 218 within [10] ) says that $X^{*}$ has the RNP if and only if the dual of every separable subspace of $X$ is separable. Moreover, Namioka/Phelps (1975, p. 741 within [18]) proved that the dual of an Asplund space has the Radon Nikodym property.

Theorem 3.4. [11] Every Asplund space and its dual admits rotund renorming.

Theorem 3.5 ([11], p. 57). If the dual norm of $X^{*}$ has LUR norm then $X$ admits an equivalent LUR norm.

Theorem 3.6. [7] Every locally uniformly rotund norm on a Banach space has the Kadec-Klee property.

Proof. Let $\mathrm{X}$ be a linear space with a locally uniformly rotund norm $\|\cdot\|$. Suppose that $G$ is open for the norm topology on $S(X)$. That means, if $x \in$ $G \bigcap S(X)$, then there $\varepsilon>0$ such that $B(x, \varepsilon):=\{y:\|y-x\| \leq \varepsilon\} \subseteq G$. We will prove that $G$ is open for the weak topology on $S(X)$. Since $\|\cdot\|$ is $\mathrm{LUR}$, there is $\delta>0$ such that for all $y \in S(X),\left\|\frac{x+y}{2}\right\|>1-\epsilon$ implies that $\|x-y\|<\varepsilon$. Now there is an $f \in X^{*}$ such that $f(x)=\|f\|=1$ by Hahn-Banach theorem for normed spaces. So $V:=\{y: f(y)>1-\delta\}=\{y: f(x)-f(y)<\delta\}$ is open for the weak topology. Moreover, for all $y \in V \cap S(X),\left\|\frac{x+y}{2}\right\| \geq \frac{1}{2}\|f\| \cdot\|x+y\|$

$$
\geq \frac{1}{2} f(x+y)=\frac{1}{2}[f(x)+f(y)]>1-\frac{\delta}{2}>1-\delta .
$$


Thus

$$
\|x-y\|<\varepsilon
$$

Therefore $y \in G$. Thus, $V \cap S(X) \subseteq G$. As $x$ is arbitrary, $G$ is open for the weak topology on $S(X)$, as $G$ is arbitrary, the norm and weak topologies agree on $S(X)$.

Its converse is shown by Troyansski in [21]. The authors proved that if $X$ has RNP (in particular, if $X$ is dual of some Asplund spaces ), then $X$ has LUR renorming iff it has kadec renorming. Haydon [11] gave examples showing that the rotundity assumption is essential for constructing trees such that $C(Y)$ has KKP but not rotund renorming.

Theorem 3.7. [7] A Banach space $X$ admits an equivalent LUR norm if and only if it has a strictly convex norm with kadec-klee property $(K K P)$.

Proof. Let a norm $\|\cdot\|$ of a Banach space $X$ which is both strictly convex and has Kadec Klee property. By Asplund Averaging procedure we combine both norms to reach at ususal goal ([4], p. 52). We have a fact: for every $x \in S_{X}$ and $\varepsilon>0$, there is a half space $H$ such that $x \in H$ and $\operatorname{diam}(H \cap B(X))<$ $\varepsilon$, i. e. each point of the new sphere is denting point. For $m \in N$, put $A_{m}=\left\{x \in B(X): \operatorname{diam}(H \bigcap B(X))>\frac{1}{m}\right.$, for all half spaces $H$ containing $x\}$, then the set $A_{m}$ is closed convex symmetric and $0 \in\left(A_{m}\right)^{0}$. So there is a Minkowski functional of $A_{m}$ say $f_{m}$, defined by $f_{m}(x)=\left\{t>0: \frac{x}{t} \in A_{m}\right\}$ which is equivalent norm on $X$. Let $a_{m}>0$ defined by $a_{m} f_{m}^{2}(x) \leq \frac{\|x\|^{2}}{2^{m}}$ for all $x \in X$. Define $|x|^{2}=\sum_{m=1}^{\infty} a_{m} f_{m}^{2}(x)+\|x\|^{2}$ for all $x \in X$, then $\|x\|^{2} \leq|x|^{2}=$ $\sum_{m=1}^{\infty} a_{m} f_{m}^{2}(x)+\|x\|^{2} \leq \sum_{m=1}^{\infty} \frac{1}{2^{m}}\|x\|^{2}+\|x\|^{2}$. Thus

$$
\|x\|^{2} \leq|x|^{2} \leq\left(\sum_{m=1}^{\infty} \frac{1}{2^{m}}+1\right)\|x\|^{2}
$$

So

$$
\|x\|^{2} \leq|x|^{2} \leq 2\|x\|^{2}\left(\text { by } \sum_{m=1}^{\infty} \frac{1}{2^{m}}=1\right) .
$$

Hence

$\|x\| \leq|x| \leq \sqrt{2}\|x\|$ for all $x \in X$. This shows that $\|\cdot\|$ and $|\cdot|$ are equivalent norms for all $x \in X$. 
Now, to show $|\cdot|$ is LUR norm on $X$. For it, take $x, x_{n} \in X$ such that

$$
2|x|^{2}+2\left|x_{n}\right|^{2}-\left|x+x_{n}\right|^{2} \rightarrow 0
$$

we have to show that $\left|x_{n}-x\right| \rightarrow 0$.

If $x=0$, then the result is clear. otherwise let $x$ is not equal to 0. Multiply $x$ and $x_{n}$ by $\frac{1}{\|x\|}$, we assume that $\|x\|=1$, and from [[7], fact 2.3, p. 45$]$ is reduced to the form

$$
2\|x\|^{2}+2\left\|x_{n}\right\|^{2}-\left\|x_{n}+x\right\|^{2} \rightarrow 0 .
$$

So we get, $\left\|x_{n}\right\| \rightarrow\|x\|=1,\left\|\frac{x_{n}+x}{2}\right\| \rightarrow 1$. replacing $x_{n}$ by $x_{n}^{\prime}=\frac{x_{n}}{\left\|x_{n}\right\|}$. We get, $\left\|x^{\prime} n\right\|=1$ and $1 \geq\left\|\frac{x+x_{n}^{\prime}}{2}\right\| \rightarrow 1$. Fix $\varepsilon>0$, find $m \in N$ with $m>\frac{2}{\varepsilon}$. since $x, x_{n}^{\prime}$ are denting points and $x, x_{n}^{\prime}$ do not belong to $A_{m}$. Because $A_{m}$ is closed $f_{m}(x)>1$ and $f_{m}\left(x_{n}^{\prime}\right)>1$. From above discuss, for large $n \in N, f_{m}\left(\frac{x_{n}^{\prime}+x}{2}\right)>1$. This shows that for these $n, \frac{x_{n}^{\prime}+x}{2}$ do not belong to $A_{m}$ and there is a half space $H$ such that $\frac{x_{n}^{\prime}+x}{2} \in H$ and $\operatorname{diam}(H \bigcap B(X)) \leq 1<\frac{\varepsilon}{2}$. Since $x, x_{n}^{\prime} \in B(X)$, then by convexity of the norm $\|\cdot\|$, we get $\left\|\frac{x_{n}^{\prime}-x}{2}\right\|<\frac{\varepsilon}{2}$, thus $\left\|x_{n}^{\prime}-x\right\|<\varepsilon$. Hence $x_{n}^{\prime} \rightarrow x$.

This shows that $\left|x_{n}-x\right| \rightarrow 0$, so $|\cdot|$ is LUR norm. Conversely, suppose that $|\cdot|$ is LUR norm on $X$, we know that LUR norm is strictly convex and Kadec-Klee renormed.

Remark. [22] It is clear that every locally uniformly convex Banach space is strictly convex, and examples are known which show that the converse is false. We show that the converse fails in a very strong sense.

Theorem 3.8. [22] Let $X$ be any separable Banach space. Then $X$ has an equivalent norm which is strictly convex but not locally uniformly convex.

Proof. Let $\left(x_{n}, f_{n}\right)$ be a Markusevic basis for $X$, with $\left\|x_{1}\right\|=1$ and $\left\{f_{n}\right\}$ bounded. Define $\||\cdot|||$ on $X$ by

$$
\left\||x \||=\left\{\sum_{n=1}^{\infty} 4^{-n}\left|f_{n}(x)\right|^{2}\right\}^{\frac{1}{2}}+\max \left\{\frac{1}{2}|| x||, \frac{1}{2}\left|f_{1}(x)\right|+\sup _{2 \leq n<\infty}\left|f_{n}(x)\right|\right\} .\right.
$$

Then $\||\cdot \||$ is weak lower semicontinuous, and so arises from an equivalent norm on $X$. The first term in the expression above ensures that $(X,\||\cdot \||)$ is strictly convex. Thus $\||\cdot|\|$ is an equivalent strictly convex norm for $X$. Note that $\left\|\left|x_{1}\left\|\left|=1,\left\|\left|x_{n} \|\right| \rightarrow 1\right.\right.\right.\right.\right.$ and $\left.\left.\left.\|\right| x_{1}+x_{n}\right\|\right| \rightarrow 2$. However $f_{1}\left(x_{n}\right) \rightarrow 0$ and $f_{1}\left(x_{1}\right)=1$, so $\left(x_{n}\right)$ does not even converge weakly to $x_{1}$. 
Final remarks: Suppose that $X$ is a space with the Radon-Nikodym property. Does $X$ admit an equivalent locally uniformly rotund (LUR) norm (and Rotund norm)? [6]

1. Let $X^{*}$ has RNP. Then $X$ is Asplund space by theorem 2.3. It is shown in (Corollary VII.1. 12, p, $266[6]$ ), that "If $X$ be an Asplund space. Then $X^{*}$ admits an LUR norm (which is not dual in general)". Recall that LUR norm implies Rotund norm and KKP. Thus the answer to this question is positive if $X$ is a dual space.

2. If a Banach space has RNP with countable dentability index, then the above question also has positive answer which is shown in [16].

3. In the special case when the Banach space $X$ enjoys the Radon-Nikodym property, it is still an open problem to decide whether $X$ has even an equivalent rotund norm or not. In that case, by the results in [17], the existence of a LUR and a Kadec equivalent norm are indeed equivalent.

4. Let us remark here that Yost and Plicko in [19] proved that the RNP does not imply the separable complementation property. Thus it is not possible any approach to the former question based on the projectional resolution of the identity; at the contrary, such an approach works for the dual case, as in [10]. We note that the following statement $([9]$, p. 208) is still true. It is unknown to this day whether each Banach space with the Radon-Nikodym property admits an equivalent strictly convex norm." We are equally ignorant about locally uniformly convex norms.

5. It is an easy exercise to show that every LUR space has this property. The following partial converse appears in [17], if a Banach space has the KreinMilman property (which is implied by RNP) and the Kadets-Klee property, then it has an equivalent locally uniformly convex norm.

Fabian and Godefroy [18] proved that every dual space with RNP has an equivalent locally uniformly convex norm, but for non-dual spaces, the question remains open. That our example does admit such a norm follows from its possession of a strong Markusevic basis [2], or by direct calculation.

Thus we summarize exactly the above open problem. In my research work no examples and no results are found the question is right.

\section{Acknowledgments}

The first author would like to express his gratitude to his $\mathrm{Ph}$. D advisor, Prof. Prakash Muni Bajracharya, for his encouragement, constant support, helpful remarks and insightful comments on this paper. I also would like to express 
my deep gratitude to UGC of Nepal for financial support. The work of third author was supported by the National Natural Science Foundation of China (11571378).

\section{References}

[1] A. Amanollah, H. Haghshenas, Some classical and recent results concerning renorming theory. Thai J. Math., 10(3) (2012), 481-495.

[2] G. A. Aleksandrov, A. N. Plichko, The connections between strong M-bases and equivalent locally uniformly convex norms in Banach spaces. (Russian) C. R. Acad. Bulgare Sci., 40(2) (1987), 15-16.

[3] M. Bachir, A. Daniilidis, A dual characterisation of the RadonNikodym property. Bull. Austral. Math. Soc., 62(3) (2000), 379-387. http://dx.doi.org/10.1017/S000497270001889X

[4] R. D. Bourgin, Geometric aspects of convex sets with the Radon-Nikodym property. Lecture Notes in Mathematics, 993. Springer-Verlag, Berlin, 1983. http://dx.doi.org/10.1007/BFb0069321

[5] J. Bourgain, A geometric characterization of the Radon-Nikodym property in Banach spaces. Compositio Math., 36(1) (1978), 3-6.

[6] R. Deville, Problemes de renormages. J. Funct. Anal. 68(2) (1986), 117-129. http://dx.doi.org/10.1016/0022-1236(86)90001-7

[7] R. Deville, G. Godefroy, V. Zizler, Smoothness and renormings in Banach spaces. Pitman Monographs and Surveys in Pure and Applied Mathematics, 64. Longman Scientific and Technical, Harlow; copublished in the United States with John Wiley and Sons, Inc., New York, 1993.

[8] J. Diestel, Geometry of Banach spaces-selected topics. Lecture Notes in Mathematics, Vol. 485. Springer-Verlag, Berlin-New York, 1975. http://dx.doi.org/10.1007/BFb0082079

[9] J. Diestel, J. J. Uhl, Vector measures. With a foreword by B. J. Pettis. Mathematical Surveys, No. 15. American Mathematical Society, Providence, R.I., 1977. http://dx.doi.org/10.1090/surv/015

[10] M. Fabian and G. Godefroy, The dual of every Asplund space admits a projectional resolution of the identity. Studia Math., 91(2) (1988), 141-151.

[11] J. Hagler, A counterexample to several questions about Banach spaces. Studia Math., 60(3) (1977), 289-308.

[12] R. G. Haydon, C. A. Rogers, A locally uniformly convex renorming for certain $C(K)$. Mathematika, 37(1) (1990), 1-8. http://dx.doi.org/10.1112/S0025579300012754

[13] R. Haydon, Locally uniformly convex norms in Banach spaces and their duals. J. Funct. Anal., 254(8) (2008), 2023-2039. http://dx.doi.org/10.1016/j.jfa.2007.11.005

[14] R. Haydon, Trees in renorming theory. Proc. London Math. Soc., 78(3) (1999), 541-584. http://dx.doi.org/10.1112/S0024611599001768

[15] R. E. Huff, Dentability and the Radon-Nikodym property. Duke Math J., 41 (1974), 111-114. 
[16] G. Lancien, Dentability indices and locally uniformly convex renormings. Rocky Mountain J. Math., 23(2) (1993), 635-647. http://dx.doi.org/10.1216/rmjm/1181072581

[17] A. Molto, J. Orihuela, S. Troyanski, and M. Valdivia, Kadec and KreinMilman properties. C. R. Acad. Sci. Paris Ser. I Math. 331(6) (2000), 459-464. http://dx.doi.org/10.1016/S0764-4442(00)01644-X

[18] A. Pietsch, History of Banach spaces and linear operators. Birkhauser Boston, Inc., Boston, MA, 2007.

[19] A. M. Plichko and D. Yost, The Radon-Nikodym property does not imply the separable complementation property. J. Funct. Anal., 180(2) (2001), 481-487. http://dx.doi.org/10.1006/jfan.2000.3689

[20] F. Sullivan, On the duality between Asplund spaces and spaces with the Radon-Nikodym property. Proc. Amer. Math. Soc., 71(1) (1978), 155C156. http://dx.doi.org/10.2307/2042240

[21] M. Talagrand, Renormages de quelques $C(K)$. Israel J. Math., 54(3) (1986), 327-334.

[22] D. Yost, M-ideals, the strong 2-ball property and some renorming theorems. Proc. Amer. Math. Soc., 81(2) (1981), 99-303. http://dx.doi.org/10.1090/S0002-9939-1981-0593475-7. 
